\title{
Concepciones sobre la realidad aumentada en el profesorado de Infantill y Primaria
}

\section{Conceptions about augmented reality in kindergarten and primary teachers}

\section{Pérez Castello, Teresa; Moreno Vera, Juan Ramón}

Teresa Pérez Castello td.perezcastello@um.es Universidad de Murcia, España

Duan Ramón Moreno Vera jr.moreno@um.es Universidad de Murcia, España

Clio \& Asociados. La historia enseñada Universidad Nacional del Litoral, Argentina

ISSN: 2362-3063

Periodicidad: Semestral

núm. 33, 2021

clio@fhuc.unl.edu.ar

\section{Recepción: 27 Julio 2021}

Aprobación: 18 Agosto 2021

\section{URL: https://doi.org/10.14409/cya.v0i33.10513}

\section{(i) (2)}

Esta obra está bajo una Licencia Creative Commons AtribuciónNoComercial-CompartirIgual 4.0 Internacional.
Resumen: La experiencia práctica presentada se propone analizar las concepciones que tiene el profesorado de Educación Infantil y Primaria sobre el uso didáctico y práctico de la Realidad Aumentada en las aulas de Ciencias Sociales. El carácter innovador de la Realidad Aumentada y su potencial de aprendizaje, ofrecen un escenario educativo único, motivador y transformador de la realidad educativa actual. Identificaremos sus posibilidades como recurso innovador y elemento clave en el desarrollo de una cultura de aprendizaje tecnológica, centrada en el área de Ciencias Sociales y valores educativos. El análisis estadístico-descriptivo de los datos se ha llevado a cabo mediante SPSS v24 y constata la importancia de ampliar la formación del profesorado en esta herramienta y la necesidad de favorecer propuestas metodológicas innovadoras que fundamentan la realidad tecnológica actual, presente y activa en las aulas de Infantil y Primaria.

Palabras clave: realidad aumentada, Ciencias Sociales, educación, tecnología educativa, enseñanza-aprendizaje.

Abstract: The practical experience presented analyzes the perception that Early-Childhood and Primary teachers have about the didactic and practical use of Augmented Reality teaching Social Sciences. The innovative nature of Augmented Reality and its learning potential offer a unique, motivating and transforming learning scenario of the current educational reality. We will identify its possibilities as an innovative resource and key element in the development of a culture of technological learning, focused on the area of Social Sciences and educational values. The statistical-descriptive analysis of the data, done through SPSS $v 24$ reflect the importance of expanding teacher training in this tool and the need to promote innovative methodological proposals that support the current, present and technological reality active in the Infant and Primary classrooms.

Keywords: augmented reality, Social Sciences, education, educational technology, teaching-learning.

\section{Introducción}

La Realidad Aumentada (RA) como enunciado, concepto y recurso didáctico ha irrumpido en las aulas de infantil y primaria, como propuesta metodológica 
innovadora y como planteamiento lúdico, ofreciendo al profesorado una herramienta accesible, de fácil manejo y cada vez más visible, en el entorno inmediato de nuestro alumnado.

La progresiva incorporación actual de las TIC en las aulas no garantiza el éxito del proceso educativo. Es necesario un compromiso por parte del colectivo docente, en el uso de tecnologías innovadoras y facilitadoras de procesos de enseñanza-aprendizaje flexibles. Somos capaces de despertar la curiosidad y motivación (Gómez-Carrasco et al., 2019 y 2020) de nuestro alumnado e iniciar el desarrollo de procesos cognitivos superiores a partir del uso adecuado de estas herramientas.

En ese sentido, "las TIC deben ser instrumentos favorecedores de aprendizajes significativos y relevantes para los estudiantes, de situaciones y experiencias que sean fuente de innovación y, por supuesto, de motivación" (Cabero et al., 2016:12).

Desde esta concepción, la escuela:

Debe ser un escenario donde los estudiantes empleen las TIC con el fin de construir su propio conocimiento, de adquirir las competencias clave necesarias para que sean buenos ciudadanos. Ciudadanos felices y activos, comprometidos y reparados para un sistema económico y cultural complejo y en permanente reinvención (...). Se trata de que los estudiantes utilicen las TIC para hacer, para compartir, para transferir y para reconstruir su propio pensamiento desde la autonomía (Cabero et al., 2016:15).

El diseño, la elaboración e introducción de materiales multimedia interactivos en la práctica diaria del aula, podrá favorecer el proceso de enseñanza-aprendizaje entre el alumnado, si centra su atención en el uso didáctico de estas herramientas, en su fuerza innovadora y en su capacidad de transformar e interpretar de forma constructiva y crítica la realidad.

Se trata de incorporar a esta práctica, experiencias innovadoras, itinerarios diversos, conocimientos culturales diversos y valoración de sus rasgos diferenciales, como elementos enriquecedores de la sociedad. En resumen, podemos favorecer la construcción de un nuevo modelo y cultura de aprendizaje, que permita al alumnado la adquisición, incorporación, interiorización y el análisis crítico y real de la información.

Coll (2008) señala que las/os docentes con una visión del proceso de enseñanza y aprendizaje más activo o constructivista, utilizan las TIC para promover actividades de exploración, trabajo autónomo y colaborativo.

Como afirman Gutiérrez et al. "es positivo ser innovador, incorporar las novedades a nuestras aulas, pero sin perder de vista que la mera incorporación de lo novedoso no lleva parejo el éxito del proceso educativo (2015:148)". Se trata por tanto de ser conscientes de la finalidad de nuestro planteamiento didáctico y del uso de las herramientas tecnológicas, como medios en sí mismas y no como fines para el logro del aprendizaje.

\section{La realidad aumentada en la enseñanza de las ciencias sociales}

La Realidad Aumentada es un concepto que combina componentes reales y virtuales que se aúnan favoreciendo la interacción, habitualmente en tiempo real, a partir de dispositivos móviles y ordenadores. Tal y como señala Azuma (1997), 
complementa la realidad presente mejorando la interacción y percepción del usuario con el mundo real.

"La RA hace referencia a la visualización directa o indirecta de elementos del mundo real combinados (o aumentados) con elementos virtuales generados por un ordenador, cuya fusión da lugar a una realidad mixta" (Cobo Romaní y Moravec, 2011:105).

Los términos de Realidad Aumentada y Realidad Virtual implican una diferenciación en su significado. Mientras que la realidad virtual hace referencia a un entorno virtual y tecnológico en el que puede existir o no interacción, en la RA nos basamos en el mundo físico para lograr la interacción con los elementos de nuestro entorno real.

La RA ofrece por tanto un gran potencial de aprendizaje y una alternativa innovadora para la comunidad educativa. En este sentido, son numerosas las posibilidades que ofrece el uso didáctico de la RA en las aulas de Infantil y Primaria:

- Permite una aplicación multidisciplinar y una generalización transversal, es decir, puede ser utilizada en cualquier área, contenido y materia educativa y en múltiples dispositivos.

- Se fundamenta en el conocimiento práctico, favoreciendo el acceso al conocimiento intuitivo, procedimental y directo de la información.

- Favorece la interiorización, adquisición y ampliación de nuevos conocimientos.

- Favorece el conocimiento dirigido y la toma de contacto inicial del alumnado con las tecnologías, mostrando el contenido didáctico de este sistema de aprendizaje, destacando que esta función complementa su sentido lúdico.

- Favorece la búsqueda de información, la ampliación de conocimientos y la complementación de la información.

- Posibilita la inmersión e incursión en el diseño de actividades que favorecen la investigación y el descubrimiento.

- Generaliza el uso didáctico y formativo de las TIC como elemento de aprendizaje y herramienta de trabajo en el aula.

- Favorece la accesibilidad de diversidad de recursos disponibles para las distintas etapas educativas.

- Se fundamenta en el juego y el entretenimiento como elemento motivador.

- Permite adaptarse a la diversidad del alumnado, a su ritmo de aprendizaje y a su propia realidad.

- Favorece el aumento de la participación directa del alumnado en el aula e incide en el desarrollo de la interactividad.

- Garantiza el proceso atencional, la curiosidad, el interés en la temática propuesta.

- Su planteamiento innovador y lúdico fomenta la creatividad en el alumnado.

- Favorece la cooperación, colaboración, interacción social y lúdica del alumnado en el aula.

- Implica un desarrollo práctico del proceso de enseñanza-aprendizaje del área de Ciencias Sociales y la educación en Valores. 
"En suma, podríamos decir que las TIC están transformando los escenarios educativos tradicionales, al tiempo que están haciendo aparecer otros nuevos" (Coll 2004:5). Aplicada esta idea a la realidad actual tecnológica, la RA se convierte en un recurso multidimensional que ofrece grandes posibilidades en el ámbito educativo y así lo demuestran las experiencias y estudios realizados hasta el momento. Se trata de una tecnología innovadora que permite mejorar la percepción del mundo real incorporando información superpuesta.

Diversas investigaciones sobre el uso pedagógico de la RA en las aulas y su relación con el aprendizaje significativo, confieren su valor educativo. Entre dichas investigaciones, destacamos el estudio de Gutiérrez et al. (2015), centrado en una investigación basada en las tecnologías emergentes actuales y su repercusión en la enseñanza de las Ciencias Sociales. Basaron su propuesta en una experiencia didáctica realizada con los/as futuros/as docentes de Ciencias Sociales de Educación Infantil y Primaria, con el objetivo de conocer su opinión sobre la Realidad Aumentada y su aplicación educativa.

Los resultados del estudio concretaron la novedad, implicación, motivación y desafío que la instrumentalización educativa de la RA supone para los estudiantes (Gutiérrez et al, 2015).

Por su parte, las conclusiones destacaron que los/as encuestados/as valoraban de forma muy positiva la apreciación, uso y utilidad de las TIC en la docencia. Se reflejaba, por tanto, la necesidad didáctica que tenían en su formación inicial como docentes y en este sentido, las TIC innovadoras favorecían la docencia, como hecho incuestionable en el desarrollo de las prácticas pedagógicas actuales.

En lo que respecta a la RA, el estudio concluye que favorece el proceso de enseñanza-aprendizaje, motiva al alumnado, facilita la comprensión de contenidos y la elaboración de materiales didácticos y actividades de aprendizaje, relativas al estudio de nuestro entorno más cercano.

Compartiendo estas aportaciones, consideramos que la RA es un recurso que permite presentar, secuenciar, organizar y explicar los contenidos curriculares del área de Ciencias Sociales de una forma atractiva, práctica e innovadora, favoreciendo el aprendizaje significativo, experiencial, vivencial, funcional y motivacional.

Desde esta perspectiva, también es necesario fomentar el uso de la RA como herramienta para la producción de contenidos, por parte del alumnado, sin embargo, somos conscientes de que se requieren múltiples condiciones para ello:

Si nos centramos en la RA como herramienta tecnológica utilizada por los alumnos para el desarrollo y creación de contenidos, los estudios son aún menos, debido principalmente a la novedad de la tecnología, la necesidad de contar con tecnologías específicas para su desarrollo, la formación que los docentes suelen tener respecto a estas tecnologías y las pocas experiencias educativas realizadas en este campo (Barroso Osuna y Gallego Pérez, 2016:119)

La RA es un concepto que conjuga elementos de la realidad más inmediata y próxima al alumnado, con elementos virtuales favoreciendo el aprendizaje, permitiendo la interacción de ambos elementos desde cualquier dispositivo tecnológico desde la propia conjunción dinámica y lúdica de realidad y ficción. Nos permite emplear el mundo físico como espacio de referencia e interactuar con elementos que forman parte de nuestro entorno más inmediato. 
Tal y como describen Cabero-Almenara y Barroso Osuna, "la RA es la combinación de información digital e información física en tiempo real a través de diferentes dispositivos tecnológicos" (2016:46).

Es un recurso innovador, lúdico y motivador favorecedor del proceso de enseñanza-aprendizaje, fundamentado en el descubrimiento y el interés que despierta entre el alumnado de las distintas etapas educativas.

Desde propuestas educativas que inciden en la importancia de la innovación tecnológica y la implementación de tecnologías educativas emergentes en las aulas, la Realidad Aumentada se concibe como un elemento clave en la promoción de nuevas estrategias de aprendizaje, junto con otros recursos como los códigos QR y la ludificación, entre otros (Rodríguez-García et al., 2019).

Por su parte, González et al. (2019), en un estudio realizado con una muestra de 220 participantes, en el que pretendía conocer las percepciones sobre la relación entre la Realidad Aumentada y la Realidad Virtual, para favorecer la Educación en Valores y el fomento de la convivencia, se obtuvieron conclusiones que vinculaban ambos conceptos. Su estudio constataba que la elaboración de apps centradas en la RA, favorecía el conocimiento de herramientas que podían servir para la Educación en Valores y la convivencia escolar.

Este estudio fundamenta uno de los ítems del cuestionario en el que pretendemos identificar el uso instrumental de la RA y la formación en valores del alumnado.

López García y Miralles en una experiencia de innovación realizada en las prácticas del Máster de formación del Profesorado de Educación Secundaria, basada en la utilización de la RA como recurso TIC básico, concluye:

Pero para que este tipo de propuestas tengan éxito a largo plazo se antoja imprescindible adoptar una actitud en consonancia con los avances del siglo XXI. El profesorado actual debe adaptarse a estos cambios en todas sus modalidades, y la rama tecnológica, en esta era de máxima digitalización, no merece menos atención, ya que es uno de los andamios que sustentan el desarrollo personal y profesional de los futuros maestros (López García y Miralles, 2018:45).

\section{Método}

\subsection{Objetivos especificos de la investigación}

En el caso de la experiencia didáctica que aquí se presenta, nos hemos centrado en la Realidad Aumentada y la concepción sobre ella que tienen los/as docentes en Infantil y Primaria. Desde esta propuesta hemos desarrollado una serie de objetivos específicos:

- O.E. 1. Identificar el conocimiento y uso práctico de la Realidad Aumentada, en el profesorado en activo y el futuro profesorado de Educación Infantil y Primaria.

- O.E.2. Analizar la realización de prácticas educativas basadas en Realidad Aumentada.

- O.E. 3. Conocer el uso de la Realidad Aumentada en relación a la educación en valores. 


\subsection{Descripción del contexto y participantes}

En lo que respecta a la muestra participante en el estudio, destacamos que se trata de dos grupos diferenciados, por un lado, profesorado en activo de un centro de Educación Infantil y Primaria de la provincia de Alicante y por otro, futuros/ as docentes matriculados/as en el Grado de Educación Infantil y Educación Primaria, de la Facultad de Educación de la Universidad de Alicante.

De los cuestionarios implementados, dos de ellos se realizaron en el curso 18-19. En estos cuestionarios participaron dos grupos formados por 14 y 41 participantes respectivamente. Otro de los cuestionarios se administró durante el curso 19-20, con una muestra total de 33 respuestas.

El estudio se ha realizado con una muestra total de 88 participantes $(n=88)$. De forma más específica:

- Cuestionario elaborado a docentes en activo, en un centro de Educación Infantil y Primaria de titularidad pública (14 participantes). En este cuestionario implementado participaron un total de 12 docentes de Educación Primaria y 2 docentes de Educación Infantil. De la muestra total de 14 participantes, 13 eran maestras y participó 1 maestro.

- Cuestionario aplicado al alumnado matriculado en el Grado de Educación Infantil, por lo tanto, futuro profesorado de esta etapa (74 participantes). De la muestra total de 74 participantes, 66 eran maestras y participaron 8 maestros.

\subsection{Instrumento, fiabilidad y procedimiento de análisis}

El método de investigación utilizado en esta experiencia didáctica, se centró en el diseño "ad hoc" e implementación de un cuestionario on-line, con 2 respuestas dicotómicas y una respuesta tipo Likert, que fue, posteriormente, analizada mediante el paquete estadístico de análisis de datos IBM SPSS 24. Esta herramienta nos permitió recopilar, de forma precisa, la información contenida en las respuestas del alumnado (y futuros docentes) y los/as docentes participantes, a través de un análisis estadístico-descriptivo de datos, tal y como se ha llevado a cabo en otras investigaciones en Didáctica de las Ciencias Sociales sobre la implementación de las prácticas docentes en el aula. El cuestionario contenía 3 bloques de ítems referidos a:

- Ítem 1: Conozco alguna aplicación (APPS) para trabajar la Realidad Aumentada en el aula.

- Ítem 2: He realizado alguna actividad durante mi formación con códigos QR y Realidad Aumentada.

- Ítem 3: Considero que el uso de las WebQuests y la Realidad Aumentada puede ayudar a instruir y formar en valores a mi alumnado.

El cuestionario utilizado en la presente investigación ha sido validado a través de la prueba del Alfa de Cronbach, que evalúa la fiabilidad interna del constructo. El resultado obtenido ha sido de 0.681 , lo que indica una aceptable fiabilidad interna de los ítems en cuestión a tener de lo especificado en otras investigaciones precedentes en la enseñanza de las Ciencias Sociales, como es el caso de Gómez- 
Carrasco et al. (2019 y 2020), que manifiesta que, valores entre 0.6 y 0.9 muestra una buena consistencia interna del instrumento.

\section{Resultados}

Para mostrar los resultados se ha dividido la muestra en dos bloques diferenciados estableciendo los siguientes apartados:

- Análisis de resultados del cuestionario aplicado a docentes en activo, en un centro de Educación Infantil y Primaria de titularidad pública (14 participantes).

- Análisis de resultados del cuestionario aplicado al alumnado matriculado en el Grado de Educación Infantil y Primaria y por lo tanto futuro profesorado de estas etapas ( 74 participantes).

A continuación, realizaremos una muestra de las tablas, con los datos de frecuencias y porcentajes de los 3 ítems analizados.

\section{Tabla 1.}

Conozco alguna aplicación (APPS) para trabajar la Realidad Aumentada en el aula.

\begin{tabular}{|c|c|c|c|c|c|}
\hline & & Frecuencia & Porcentaje & $\begin{array}{l}\text { Porcentaje } \\
\text { válido }\end{array}$ & $\begin{array}{l}\text { Porcentaje } \\
\text { acumulado }\end{array}$ \\
\hline \multirow[t]{4}{*}{ Válido } & NO & 10 & 71,4 & 71,4 & 71,4 \\
\hline & NS INC & 1 & 7,1 & 7,1 & 78,6 \\
\hline & SI & 3 & 21,4 & 21,4 & 100,0 \\
\hline & Total & 14 & 100,0 & 100,0 & \\
\hline
\end{tabular}

Elaboración propia.

Los resultados de la Tabla 1, relativos al conocimiento por parte de los/ as docentes en activo, de aplicaciones de RA en el aula reflejan que el 71,4\% desconoce las posibilidades de este recurso frente al $21 \%$ que si identifica su uso y aplicación didáctica y práctica en el aula.

\section{Tabla 2.}

He realizado alguna actividad en el Grado Universitario de Educación Infantil/Primaria con códigos QR y Realidad Aumentada.

\begin{tabular}{|c|c|c|c|c|c|}
\hline & & Frecuencia & Porcentaje & $\begin{array}{l}\text { Porcentaje } \\
\text { válido }\end{array}$ & $\begin{array}{l}\text { Porcentaje } \\
\text { acumulado }\end{array}$ \\
\hline \multirow[t]{3}{*}{ Válido } & 1,00 & 1 & 7,1 & 7,1 & 7,1 \\
\hline & 2,00 & 13 & 92,9 & 92,9 & 100,0 \\
\hline & Total & 14 & 100,0 & 100,0 & \\
\hline
\end{tabular}


Los resultados de la Tabla 2 muestran que el 92,9\% del profesorado en activo participante no ha realizado alguna actividad relativa a RA en su formación académica frente a un $7,1 \%$ que sí lo ha hecho.

\section{Tabla 3.}

Considero que el uso de las WebQuests y la Realidad Aumentada puede ayudar a instruir y formar en valores a mi alumnado.

Tabla 3. Considero que el uso de las WebQuests y la Realidad Aumentada puede ayudar a instruir y formar en valores a mi alumnado.

\begin{tabular}{lll|l|l|l} 
& & & & $\begin{array}{l}\text { Porcentaje } \\
\text { válido }\end{array}$ & $\begin{array}{l}\text { Porcentaje } \\
\text { acumulado }\end{array}$ \\
\hline Válido & 2,00 & 2 & 14,3 & 15,4 & 15,4 \\
& 3,00 & 5 & 35,7 & 38,5 & 53,8 \\
\hline & 4,00 & 3 & 21,4 & 23,1 & 76,9 \\
\hline & 5,00 & 3 & 21,4 & 23,1 & 100,0 \\
\hline Perdidos & Total & 13 & 92,9 & 100,0 & \\
Total & & 14 & 100,0 & & \\
\hline
\end{tabular}

Elaboración propia.

Los resultados de la Tabla 3 reflejan que el 15,4\% de la muestra participante se manifiesta en desacuerdo en relación al uso de las WebQuests como recurso para el desarrollo de la educación en Valores en las aulas frente al 23\% que está totalmente de acuerdo en el uso de estas herramientas en la interiorización y adquisición de valores en las aulas.

\section{Tabla 4}

Conozco alguna aplicación (APPS) para trabajar la Realidad Aumentada en el aula.

Tabla 4. Conozco alguna aplicación (APPS) para trabajar la Realidad Aumentada en el aula.

\begin{tabular}{lll|l|l|l} 
& & Frecuencia & Porcentaje & $\begin{array}{l}\text { Porcentaje } \\
\text { válido }\end{array}$ & $\begin{array}{l}\text { Porcentaje } \\
\text { acumulado }\end{array}$ \\
\hline Válido & NO & 30 & 40,5 & 40,5 & 40,5 \\
\cline { 2 - 5 } & NS/NC & 2 & 2,7 & 2,7 & 43,2 \\
\hline & NS/NC & 7 & 9,5 & 9,5 & 52,7 \\
\hline & Sí & 35 & 47,3 & 47,3 & 100,0 \\
\hline & Total & 74 & 100,0 & 100,0 & \\
\hline
\end{tabular}

Elaboración propia.

Los resultados de la tabla 4 relativos al análisis estadístico de los datos correspondientes al ítem 1, reflejan que el 40,5\% del futuro profesorado de Educación participante desconoce aplicaciones de RA de uso didáctico para el trabajo en las aulas, frente a un $47,3 \%$ que presenta conocimiento del recurso. 


\section{Tabla 5.}

He realizado alguna actividad en el Grado Universitario de Educación Infantil/Primaria con códigos QR y Realidad Aumentada.

\begin{tabular}{|c|c|c|c|c|c|}
\hline & & Frecuencia & Porcentaje & $\begin{array}{l}\text { Porcentaje } \\
\text { válido }\end{array}$ & $\begin{array}{l}\text { Porcentaje } \\
\text { acumulado }\end{array}$ \\
\hline \multirow[t]{4}{*}{ Válido } & NO & 33 & 44,6 & 44,6 & 44,6 \\
\hline & NS INC & 1 & 1,4 & 1,4 & 45,9 \\
\hline & SI & 40 & 54,1 & 54,1 & 100,0 \\
\hline & Total & 74 & 100,0 & 100,0 & \\
\hline
\end{tabular}

Fuente: Elaboración propia.

Los resultados de la Tabla 5 reflejan que un 44,6\% del futuro profesorado no ha realizado actividades vinculadas a RA dentro del ámbito académico del grado universitario respecto a un $54,1 \%$ que ha empleado este recurso.

\section{Tabla 6.}

Considero que el uso de las WebQuests y la Realidad Aumentada puede ayudar a instruir y formar en valores a mi alumnado

\begin{tabular}{|c|c|c|c|c|c|}
\hline & & Frecuencia & Porcentaje & $\begin{array}{l}\text { Porcentaje } \\
\text { válido }\end{array}$ & $\begin{array}{l}\text { Porcentaje } \\
\text { acumulado }\end{array}$ \\
\hline \multirow{5}{*}{ Válido } & 2,00 & 4 & 5,4 & 5,5 & 5,5 \\
\hline & 3,00 & 16 & 21,6 & 21,9 & 27,4 \\
\hline & 4,00 & 24 & 32,4 & 32,9 & 60,3 \\
\hline & 5,00 & 29 & 39,2 & 39,7 & 100,0 \\
\hline & Total & 73 & 98,6 & 100,0 & \\
\hline Perdidos & Sistema & 1 & 1,4 & & \\
\hline Total & & 74 & 100,0 & & \\
\hline
\end{tabular}

Elaboración propia.

Por último, los resultados de la Tabla 6 indican que el 39,7\% de los futuros docentes participantes en el estudio están muy de acuerdo con la conceptualización de la RA y la WQ como recursos tecnológicos instructivos y formativos de valores en el aula, frente a un 5,5\%. En el diseño de este ítem hemos utilizado la Escala de Likert con el objetivo de obtener información sobre el grado de conformidad del alumnado participante en el estudio.

\section{Discusión y conclusiones}

Los resultados obtenidos en este estudio, suponen la respuesta a los objetivos específicos que nos habíamos planteado al inicio de la investigación y, por tanto, ayudan a conocer la meta principal que era la de analizar cuáles son las concepciones que los docentes de Educación Infantil y Primaria tienen sobre la realidad aumentada en la enseñanza de las Ciencias Sociales.

Para ello, realizaremos una reflexión sobre las conclusiones obtenidas en relación con los sub-objetivos que nos planteábamos investigar al iniciar este 
estudio. Se ha realizado un análisis de resultados de forma diferenciada en los dos bloques inicialmente descritos, por un lado, el profesorado en activo y, por otro, el futuro profesorado de Infantil y Primaria.

Por lo que respecta al objetivo específico 1, los resultados del cuestionario aplicado al profesorado en activo, muestran que, en la actualidad docente, el recurso y todas sus potencialidades siguen siendo una tarea importante a desarrollar en la práctica educativa, puesto que la mayoría de docentes admiten, en un $71,4 \%$ de los casos, que no conocen aplicaciones para trabajar la RA en el aula. Desde esta perspectiva, los resultados vinculados a este ítem, destacan la necesidad de favorecer una mayor capacitación en TIC del profesorado actual, en su formación inicial y permanente, con el objetivo de facilitar y favorecer la incorporación de la RA a la actividad diaria del aula, tal y como indican estudios previos como los de Cózar et al. (2015).

Los resultados obtenidos en el futuro profesorado corroboran la necesidad de incidir en un desarrollo de la competencia digital con bases educativas y didácticas, así como en la formación inicial del futuro profesorado de Educación Infantil sobre las posibilidades de este recurso.

En relación al objetivo específico 2, los resultados relativos al profesorado en activo ofrecen una visión muy representativa de la necesidad de implementar, dar a conocer y ofrecer propuestas vinculadas a la Realidad Aumentada, como elemento favorecedor del aprendizaje y la vinculación del alumnado de Infantil y Primaria a la realidad y comprensión del medio que le rodea. Estos resultados indican que la implementación del recurso con uso didáctico se ha iniciado, aunque no se ha consolidado, a pesar de las múltiples posibilidades que ofrece en el ámbito educativo, como elemento innovador de aprendizaje, ya que, como indican Fuentes et al (2019) la competencia digital es un factor clave en el desarrollo de metodologías activas en el aula.

Los resultados obtenidos en el futuro profesorado, reflejan que se trata de un recurso actual, que sigue formando parte de las prácticas de clase y las programaciones didácticas de educación superior. De hecho, existe una diferencia significativa en el uso de la Realidad Aumentada, en la formación universitaria del profesorado que actualmente se encuentra en activo, respecto a la formación curricular del alumnado que cursa el grado universitario en el momento de la administración del cuestionario. Este hecho refleja la importancia que se le otorga a la competencia digital en los actuales planes de estudio y la forma en la que la RA va abriéndose camino entre las propuestas más innovadoras.

Por último, con respecto al objetivo específico 3, los resultados obtenidos en el profesorado en activo, ofrecen un análisis de la importancia de fomentar, por un lado, la educación en Valores a través de las TIC y, por otro, el uso didáctico de la RA como propuesta innovadora y herramienta imprescindible en el aprendizaje de valores.

En relación al futuro profesorado de Infantil y Primaria, los resultados indican que identifican y reconocen el uso didáctico y el valor pedagógico de las WebQuests y la RA en la enseñanza-aprendizaje de valores en el aula. De hecho, se constata que la conceptualización del recurso de RA y su vinculación al aprendizaje de valores está iniciada entre el futuro profesorado de Educación Infantil y Primaria, respecto al profesorado en activo, cuyo binomio de conceptos queda bastante más lejos de su realidad docente. 
Finalizaremos el apartado con una serie de conclusiones generales:

- Analizar las diversas propuestas didácticas centradas en su uso, permitirá el diseño de nuevas metodologías favorecedoras de la interiorización y adquisición del aprendizaje para la obtención de mejores resultados académicos entre el alumnado.

- Incidir en la necesidad de plantear y favorecer estudios y experiencias innovadoras, que profundicen en la identificación de las múltiples posibilidades, acciones y aportaciones educativas que ofrece la RA.

- Emplear y favorecer el diseño de las programaciones didácticas empleando este recurso, partir de los objetivos didácticos de nuestra programación de aula, considerar las características y aspectos diferenciales de nuestro alumnado, partir de los medios, recursos e infraestructura que ofrece nuestro centro educativo, entre otros, son aspectos que favorecerán el uso didáctico y pedagógico de la RA.

\section{Referencias}

Azuma, R. T. (1997). A survey of augmented reality. Presence: Teleoperators \& Virtual Environments, 6(4), 355-385.

Barroso Osuna, J.; Gallego Pérez, O. M. (2016). La realidad aumentada y su aplicación en la educación superior. Revista Caribeña de Investigación Educativa, 1, 111-124.

Cabero Almenara, J.; Barroso Osuna, J. M. (2016). Posibilidades educativas de la Realidad Aumentada. Journal of New Approaches in Educational Research, 5(1), 46-52.

Cabero Almenara, J.; Leiva Olivencia, J. J.; Moreno Martínez, N. M.; Barroso Osuna, J.; Meneses López, E. (2016). Realidad aumentada y educación: Innovación en contextosformativos. Octaedro.

Cobo Romaní, C.; Moravec, J. W. (2011). Aprendizaje invisible. Hacia una nueva ecología de la educación. Col.lecció Transmedia XXI. Laboratori de Mitjans Interactius. Publicacions i Edicions de la Universitat de Barcelona.

Coll, C. (2004). Psicología de la educación y prácticas educativas mediadas por las tecnologías de la información y la comunicación. Una mirada constructivista. Sinéctica, Revista Electrónica de Educación, 25, 1-24.

Coll, C. (2008). Aprender y enseñar con las TIC: expectativas, realidad y potencialidades. Boletín de la institución libre de enseñanza, 72(1), 7-40.

Cózar, R.; De Moya, M. V.; Hernández, J. A.; Hernández, J. R. (2015). Tecnologías emergentes para la enseñanza de las ciencias sociales. Una experiencia con el uso de realidad aumentada en la formación inicial de maestros. Digital Education Review, 27, 138-153.

Fuentes, A.; López, J.; Pozo, S. (2019). Análisis de la competencia digital docente. Factor clave en el desempeño de pedagogías activas con realidad aumentada. REICE: revista iberoamericana sobre calidad, eficacia y cambio en Educación, 17(2), 27-42.

Gómez-Carrasco, C. J.; Monteagudo, J.; Moreno-Vera, J. R.; Sáinz, M. (2019). Effects of a Gamification and Flipped-Classroom Program for Teachers in Training on Motivation and Learning Perception. Education Sciences, 9, 299. 
Gómez-Carrasco, C. J.; Monteagudo-Fernández, J.; Moreno-Vera, J. R.; Sainz-Gómez, M. (2020). Evaluation of a gamification and flipped-classroom program used in teacher training: perception of learning and outcome. PLOS ONE, 15(10).

González Alonso, F.; Castaño Calle, R.; De Castro Hernández, R. M.; Escudero Vidal, J. (2019). Realidad Aumentada y Virtual para la Educación en Valores y la convivencia escolar. Comunicación y Pedagogía, 317-318, s/d.

Gutiérrez, R. C.; de Moya Martínez, M. D. V.; Bravo, J. A. H.; Bravo, J. R. H. (2015). Tecnologías emergentes para la enseñanza de las Ciencias Sociales.: Una experiencia con el uso de Realidad Aumentada en la formación inicial de maestros. Digital Education Review, 27, 138-153.

López-García, A.; Miralles Martínez, P. (2018). La realidad aumentada en la formación del profesorado. Una experiencia en las prácticas del Máster de Profesorado de Enseñanza Secundaria. Campus Virtuales, 7(2), 39-46.

Rodríguez-García, A. M.; Hinojo-Lucena, F. J.; Ágreda-Montoro, M. (2019). Diseño e implementación de una experiencia para trabajar la interculturalidad en Educación Infantil a través de realidad aumentada y códigos QR. Educar, 55(1), 59-77. 